\title{
Special Section Guest Editorial: Advances in Terahertz and Infrared Optoelectronics
}

\author{
Irina N. Dolganova, ${ }^{\text {a,b }}$ Dmitry S. Ponomarev, ${ }^{\text {,d }}$ Igor E. Spektor, ${ }^{d}$ \\ Michael S. Shur, ${ }^{\mathrm{e}}$ Peter S. Timashev, ${ }^{\mathrm{f}}$ and Valery V. Tuchin ${ }^{\mathrm{g}, \mathrm{h}, \mathrm{i}}$ \\ ${ }^{a}$ Institute of Solid State Physics of the Russian Academy of Sciences, \\ Chernogolovka, Russia \\ ${ }^{\mathrm{b}}$ Bauman Moscow State Technical University, Moscow, Russia \\ ${ }^{c}$ Institute of Ultra-High-Frequency Semiconductor Electronics of the Russian Academy \\ of Sciences, Moscow, Russia \\ ${ }^{d}$ Prokhorov General Physics Institute of the Russian Academy of Sciences, Moscow, Russia \\ ${ }^{e}$ Rensselaer Polytechnic Institute, Troy, New York, United States \\ ${ }^{\text {f }}$ Sechenov University, Institute for Regenerative Medicine, Moscow, Russia \\ ${ }^{9}$ Saratov State University, Science Medical Center, Saratov, Russia \\ ${ }^{\mathrm{h}}$ Institute of Precision Mechanics and Control of the Russian Academy of Sciences, \\ Saratov, Russia \\ ${ }^{i}$ National Research Tomsk State University, Tomsk, Russia
}

This special section presents recent results in the field of infrared (IR) and terahertz (THz) optoelectronics, which have been developing rapidly over the past decades. The progress of IR and $\mathrm{THz}$ technologies affects a variety of fundamental problems in material science, astrophysics, chemistry and biology, medical diagnosis, and high-resolution and multispectral imaging modalities.

Novel and improved IR and THz sources, detectors, and optical components enabled for filtering and waveguiding have revolutionized the IR and especially $\mathrm{THz}$ technology, where the problem of limited efficiency and high cost still need to be solved. The emergence of THz and IR quantum-cascade lasers, uncooled and multi-element detectors, novel types of photoconductive antennas, low-loss waveguides, advanced metamaterials have a potential for addressing these problems and, in turn, spawn further applications of $\mathrm{THz}$ and IR optoelectronics, including near-field and real-time imaging and high-resolution microscopy. The increased power of novel sources poses a question of the THz safety and dosimetry.

The special section is focused on cutting-edge fundamental and applied trends including novel materials, the interaction of $\mathrm{THz}$ and IR radiation with biological tissues, THz quantum-cascade lasers, nonlinear effects and optical light confinement in media, $\mathrm{THz}$ and IR spectroscopic and imaging systems, and advanced THz sources.

Ryzhii et al. proposed far-IR photodetectors using the graphene nanoribbon arrays and the black phosphorus (BP) base layers as the photosensitive element. The detector operation involves the interband photogeneration of the electron-hole pairs in the graphene nanoribbon array followed by the tunneling injection of either electrons or holes into a wide gap BP base layer. The detector is predicted to have a rather short response time.

Kruchunina et al. applied THz spectroscopy and dielectrophoresis for the studies of the erythrocytes of the patients with diffuse liver diseases with applications to diagnostics and differentiation of liver fibrosis. The authors obtained strong correlations of the $\mathrm{THz}$ spectroscopy indices with electrical and viscoelastic parameters of red blood cells. They also showed that the increase in the degree of liver fibrosis is associated with an increase in the number of deformed cells prone to aggregation and destruction.

Dorofeev et al. reported on the role of the tangential electric field component to the $\mathrm{THz}$ jet and the curved waves that could be produced when a light wave is passing through a dielectric cuboid or a sphere. The authors showed that the magnitude of the power flux density after passing the object is higher in a cuboid, but the focusing characteristics are better in a sphere. They

(C) 2021 Society of Photo-Optical Instrumentation Engineers (SPIE) 
also demonstrated that the screen influence is minimized when the screen is placed in the shadow of the cuboid. The terajet effect was also applied to improve the characteristics of the pointcontact detector.

Minin et al. demonstrated the enhancement of the detector's sensitivity of $6 \mathrm{~dB}$ with the 1.5 fold decrease of the noise-equivalent power. The results could be used to reduce the size and increase the sensitivity of various $\mathrm{THz}$ systems, including imaging setups for applications in medicine, biology, and astronomy.

The growing interest in $\mathrm{HgCdTe}$ quantum well heterostructures has driven the development of mid-IR semiconductor emitters. This spectrum range has a paramount practical importance because it contains absorption lines of common pollutant gases. Fadeev et al. demonstrated the stimulated emission from the $\mathrm{HgCdTe}$ heterostructures at room temperature in the vicinity of at least $2.5 \mu \mathrm{m}$. Rumyantsev et al. studied the temperature-driven photoconductivity spectra in $\mathrm{HgCdTe}$ thin films and showed the absorption edge steepness in narrow gap $\mathrm{HgCdTe}$ epilayers approaches the fundamental limit. Importantly, they revealed that the "Urbach" energy is much less than the threshold energy of the Auger recombination. This result suggests that the operating wavelengths beyond $20 \mu \mathrm{m}$ are feasible for optoelectronic devices based on $\mathrm{HgCdTe}$ structures.

$\mathrm{Li}$ et al. studied the carrier transport mechanism of $\mathrm{MoS}_{2}$ using $\mathrm{THz}$ time-domain spectroscopy. The authors found the multilayer $\mathrm{MoS}_{2}$ exhibits higher conductivity and mobility compared to the monolayer $\mathrm{MoS}_{2}$. To analyze the experimental results, they also estimated the optical conductivity using the Drude and Drude-Smith models.

Ismagilov et al. proposed the liquid jet-based broadband THz emitter. The system is based on the trapezium-shaped slit-type nozzle, whose output is confined by two parallel blades. The authors have studied the optical-to-THz conversion efficiency for various liquids and demonstrated efficiency of $0.1 \%$ using $\alpha$-pinene double-pulse excitation.

Katyba et al. developed the THz scanning-probe near-field optical microscope operating in transmission mode and using a flexible sapphire fiber. The resolution of the microscope depends on the guided mode polarization with respect to the image plane but always remains strongly subwavelength, overcoming the Abbe diffraction limit of $0.5 \lambda$. Such a subwavelength, highenergy efficiency sapphire-fiber-based $\mathrm{THz}$ microscope with a flexible design will find many applications in $\mathrm{THz}$ fundamental research and applied technology.

Shiryaev et al. explored the potential for post-acceleration of relativistic electrons in vacuum using monocyclic $\mathrm{THz}$ electromagnetic pulses augmented by constant magnetic fields. The authors showed that such an approach could provide a viable alternative to the conventional laser accelerator designs employing extremely powerful laser systems.

The development of novel materials is the focus of two papers of the section. Kuznetsov et al. studied the complex conductivity of the topological insulator (TI) $\mathrm{Bi}_{2-\mathrm{x}} \mathrm{Sb}_{\mathrm{x}} \mathrm{Te}_{3-\mathrm{y}} \mathrm{Se}_{\mathrm{y}}$ with various thicknesses and chemical compositions. The authors demonstrated the very first experimental observation of the decrease in conductivity of the $\mathrm{TI}$ in the $\mathrm{THz}$ frequency range as the chemical composition approaches the Ren's curve. Buryakov et al. studied the generation of elliptically polarized THz radiation from the BP crystallites. They observed the saturation in the dependence of the $\mathrm{THz}$ intensity on the intensity of the incident optical pump for the fluences above $6 \mathrm{~mJ} / \mathrm{cm}^{2}$.

Dubinov et al. used the InP:Fe-based waveguide frequency doubler for obtaining sub- $\mathrm{THz}$ emission. The authors showed that an optimal configuration could provide conversion efficiencies up to $4.5 \%$ under a moderate power of $1 \mathrm{~kW}$ at the fundamental frequency.

Bulgakova et al. demonstrated the generation of $\mathrm{THz}$ radiation using a $\mathrm{ZnGeP}_{2}$-based largeaperture photoconductive antenna (PCA). The authors measured the THz pulse energy dependence on the optical pump energy, and estimated the saturation fluence and carrier mobility of the PCA.

Fufurin et al. proposed numerical techniques for the IR spectra analysis of organic and inorganic volatile compounds for biomedical applications. They demonstrated the possibility of using a combination of machine and deep learning for the spectral analysis for multicomponent gas mixtures. The authors showed that neural networks trained on synthetic spectra can recognize synthetic gas mixtures and experimental individual gaseous substances.

Liu et al. described the multisegment simulation program and showed that $\mathrm{THz}$ spectrometers using plasmonic field-effect transistors (TeraFETs) could be used for the frequency to digital 
conversion. Their simulations predicted the crossover frequency which is sensitive to the channel length and nearly insensitive to the gate bias in the weak and moderate inversion regions, while the operation frequency of the frequency-to-digital converters using Si TeraFETs could vary from $110 \mathrm{GHz}$ up to $4 \mathrm{THz}$ for the TeraFETs with feature sizes from 20 to $130 \mathrm{~nm}$.

Sobolev et al. described the parameters extraction for the lumped element network representing resonant tunneling diodes. The method is based on the on-chip reflection coefficient measurements in a wide frequency range from $1 \mathrm{kHz}$ up to $60 \mathrm{GHz}$ in combination with differential resistance measurements. They obtained a reasonable agreement between the experimental curves and the curves calculated from the equivalent lumped network.

Lobanov et al. demonstrated the time-resolved measurements of light-current characteristic and mode competition in pulsed THz quantum cascade lasers (QCLs) used as THz emitters. The detection of $\mathrm{THz}$ pulses was carried out using an $\mathrm{NbN}$ superconducting hot-electron bolometer while the laser bias current was swept during a single driving pulse. The developed technique could be used for rapid characterization of pulsed THz QCLs.

Finally, Iomdina et al. proposed a voxel processing algorithm of optical coherence tomography angiography (OCTA) images to increase the accuracy of quantitative assessment of retinal and choroidal blood vessels volume. The authors demonstrated the efficiency of the algorithm by using it for determining the volumetric flow index ratio of the vascular network in the choroid and the total vascular network across the retinal and choroidal layer.

The guest editors would like to thank all contributors, the reviewers, and the Optical Engineering editorial staff for their great work and help during the preparation of this special section.

\section{Acknowledgments}

The work of guest editors I.N. Dolganova and D.S. Ponomarev was supported by a grant of RSF, \# 19-79-10212 and \# 18-79-10195, respectively. The work of guest editor V.V. Tuchin was supported by a grant of RFBR \# 18-52-16025. Michael Shur acknowledges the support from the University of Utah and Brown University. 